\title{
Crohn's Disease Manifesting as a Duodenal Obstruction: An Unusual Case
}

\author{
Jamil Shah ${ }^{\mathrm{a}, \mathrm{f}}$, Denzil Etienne ${ }^{\mathrm{b}}$, Madhavi Reddy ${ }^{\mathrm{b}}$, Jiten P. Kothadia ${ }^{\mathrm{c}}$, \\ Abul Shahidullah ${ }^{\mathrm{d}}$, A.A.M. Abdullahel Baqui
}

\begin{abstract}
We describe a case of a 31-year-old man who presented with a 3-year history of worsening upper abdominal pain, nausea, and vomiting: symptoms that were resistant to medical treatment and unexplained despite a thorough diagnostic evaluation. Then, an upper gastrointestinal series with small bowel follow-through showed proximal duodenal dilation and distal decompression of the duodenum, suggestive of a partial duodenal obstruction. An abdominal computed tomography (CT) scan revealed a transition point in the distal duodenum. At surgery, a segmental resection of the distal duodenum with a duodenojejunal anastomosis was performed. Histopathologic examination of the specimen revealed Crohn's disease. Therefore, making the diagnosis of duodenal obstruction has significant clinical implications and, in the setting of Crohn's disease, is evidence of an underlying intestinal stricture, stenotic area, or adhesion.
\end{abstract}

Keywords: Duodenal obstruction; Bowel obstruction; SBO; Crohn's disease; Inflammatory bowel disease; IBD

\section{Introduction}

Crohn's disease is a chronic inflammatory disorder that is char-

Manuscript submitted October 21, 2018, accepted November 8, 2018

aDivision of Gastroenterology and Hepatology, Department of Medicine, Rutgers New Jersey Medical School, University Hospital, 185 South Orange Avenue, Medical Science Building, Room H-538, Newark, NJ 07103, USA ${ }^{b}$ Division of Gastroenterology and Hepatology, Department of Internal Medicine, The Brooklyn Hospital Center, Academic Affiliate of The Icahn School of Medicine at Mount Sinai, Clinical Affiliate of The Mount Sinai Hospital, 121 Dekalb Avenue, Brooklyn, NY 11201, USA

'Division of Transplant Medicine, Integris Nazih Zuhdi Transplant Institute, 3300 NW Expressway, Oklahoma City, OK 73112, USA

${ }^{\mathrm{d}}$ Department of Medicine, Henry J. Carter Specialty Hospital and Nursing Facility, 1752 Park Avenue, New York, NY 10035, USA

eDepartment of Anatomic Pathology, The Brooklyn Hospital Center, Academic Affiliate of The Icahn School of Medicine at Mount Sinai, Clinical Affiliate of The Mount Sinai Hospital, 121 Dekalb Avenue, Brooklyn, NY 11201, USA ${ }^{f}$ Corresponding Author: Jamil Shah, 899 Woodmere Drive, Valley Stream, NY 11581, USA.Email: jshahid00@gmail.com.

doi: https://doi.org/10.14740/gr1105 acterized by transmural inflammation of the gastrointestinal tract. The condition may affect any part of the digestive tract from the mouth to the perianal area. The clinical manifestations of Crohn's disease vary, and patients can experience symptoms for several years prior to diagnosis. The hallmarks of the disease are abdominal cramping and pain with prolonged diarrhea (with or without gross bleeding), weight loss, fever, anemia, fatigue, and malnutrition [1]. Among the lesser known clinical manifestations is that of small bowel obstruction (SBO), which is associated with moderate-to-severe disease [2]. In the acute setting, patients present with nausea, vomiting, abdominal pain and inability to pass flatus or stool. Typically, such patients with SBO have jejunal, terminal ileum, or ileocecal involvement [2]. That our patient had isolated involvement of the duodenum and, furthermore, no additional intestinal or extraintestinal manifestations is unusual and likely contributed to the delayed diagnosis of Crohn's disease.

\section{Case Report}

A 31-year-old man, a recent immigrant to the United States from Bangladesh with no significant past medical history, presented to a primary care physician with a 3-year history of recurrent episodes of upper abdominal discomfort, nausea, and intermittent non-bloody, bilious vomiting. He was treated in the past with proton-pump inhibitors (PPIs) and antiemetic medications. Initially, his symptoms improved with medical treatment, but, gradually, they increased in frequency and severity. He denied any recent changes to his diet. He reported that no one in his household had experienced similar symptoms or become ill. He denied any recent fevers, chills, diarrhea, constipation, blood in the stool, black stools, or any other symptoms. He denied ever undergoing any surgeries. He denied ever smoking cigarettes, drinking alcohol, or using any illicit substances. He denied taking any additional medications (other than PPIs, antacids, and antiemetic agents) or any complementary, alternative, or herbal medications. He denied any allergies.

The patient was afebrile (temperature $37{ }^{\circ} \mathrm{C}$ ) and hemodynamically stable (pulse rate 88 beats per minute, blood pressure 105/60 $\mathrm{mm} \mathrm{Hg}$ ). On physical exam, he was a slender male, appearing his stated age, neatly dressed and groomed, in mild distress. The abdomen was soft and not distended. There was mild epigastric tenderness. There was no rebound tenderness, 


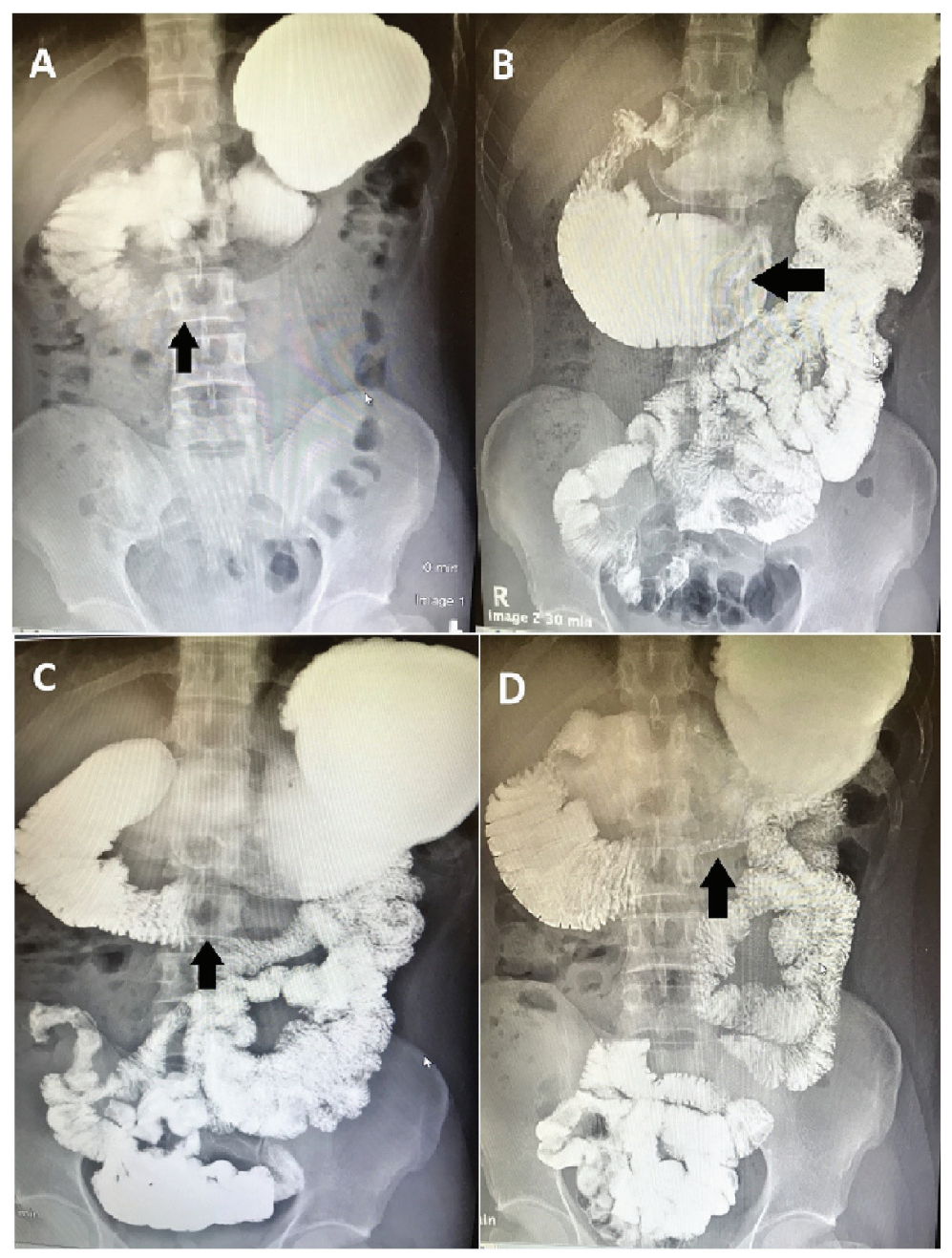

Figure 1. (A-D) Upper gastrointestinal series with small bowel follow-through. Oral contrast was administered. Note the distended stomach and proximal duodenum as well as the narrow distal duodenum. There is abnormal dilation of the third part of the duodenum, measuring $7.1 \mathrm{~cm}$ in diameter, as well as delayed passage of contrast into the distal small bowel: features suggestive of a partial distal duodenal obstruction.

guarding, or rigidity. Normoactive bowel sounds were present.

Initial laboratory testing was notable for hemoglobin of $12.3 \mathrm{~g} / \mathrm{dL}(13.5$ - $17.5 \mathrm{~g} / \mathrm{dL})$ with an unknown baseline. He had a mean corpuscular volume (MCV) of $89.7 \mathrm{fL}(80-96$ $\mathrm{fL}$ ) and a red cell distribution width (RDW) of 13.3\% (11.5$14.5 \%$ ). Also, there was mild elevation of alanine aminotransferase (ALT) $(47 \mathrm{U} / \mathrm{L}$; normal $<30 \mathrm{U} / \mathrm{L}$ for males). The serum electrolytes were within normal limits. He was referred to a gastroenterologist for further evaluation.

The patient underwent an esophagogastroduodenoscopy (EGD). He was diagnosed with H. pylori gastritis by histology and was treated with 14 days of first-line therapy with clarithromycin, amoxicillin and a PPI. However, his symptoms did not improve. Subsequently, he visited the emergency department (ED) twice with no improvement. At the second ED visit, he was given a follow-up appointment in the gastroenterology clinic. He underwent another EGD, which was negative and with no $H$. pylori detected. He then underwent a colonoscopy, which was negative. His condition worsened, and he began to experience postprandial fullness and vomiting after each meal. His appetite worsened and he went on to lose roughly 10 pounds over the next 2 weeks.

The patient visited a third gastroenterologist, who sent him for an upper gastrointestinal series with small bowel follow-through (Fig. 1). The test showed abnormal dilation of the proximal duodenum measuring $7.1 \mathrm{~cm}$ in diameter. Then, an abdominal computed tomography (CT) scan with oral contrast was performed and showed marked dilation of the second and third parts of the duodenum as well as marked narrowing of the distal third and fourth parts, which was consistent with highgrade partial small bowel obstruction at the distal duodenum (Fig. 2). He was sent to the ED and admitted to the hospital.

In the hospital, a small bowel enteroscopy was performed. A large ulcerated infiltrative mass was discovered in the fourth part of the duodenum (Fig. 3). It was circumferential and partially obstructing. The lumen could not be visualized and the scope did not pass through. The duodenal mucosa was characterized by diffuse, severe mucosal changes with congestion, 


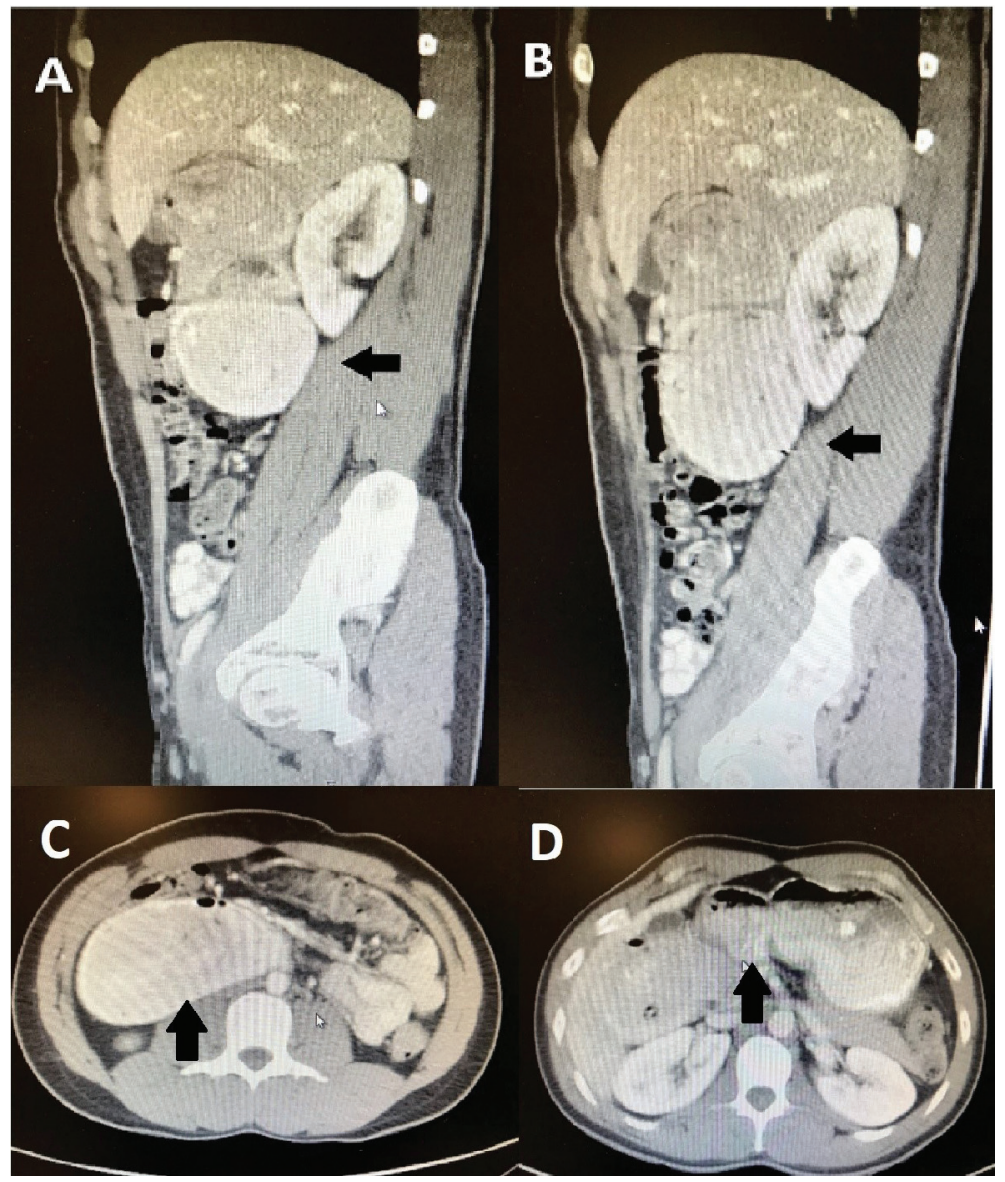

Figure 2. (A-D) Abdominal CT scan. Oral contrast was administered. The patient was discovered to have marked dilation of the second and third parts of the duodenum as well as marked narrowing of the distal third and fourth parts, which was consistent with high-grade partial small bowel obstruction at the distal duodenum.

erythema, erosions, friability, granularity, nodularity, and ulceration. Multiple biopsies were performed, and the histology showed mucosal ulceration with chronic inflammatory cells and non-caseating granulomas.

Due to this high-grade partial small bowel obstruction, the patient was sent to the operating room and a segmental resection of the distal duodenum with a duodenojejunal anastomosis was performed. The pathology report showed a narrow area of duodenum with mucosal ulceration, submucosal non-caseating granulomatous inflammation, and hypertrophic muscularis propria infiltrated by chronic inflammatory cells (Fig. 4). There was cellular spindle cell proliferation with features suggestive of fibrosis. An Acid-Fast stain and a Gomori Methenamine Silver (GMS) stain were performed and negative for mycobacterial and fungal organisms, respectively. Intestinal tuberculosis was excluded as a diagnosis with a negative interferon-gamma release assay (i.e., QuantiFERON-TB Gold test). Subsequently, a serum calcium level and a serum angiotensin-converting enzyme (ACE) level were sent to exclude sarcoidosis as a diagnosis and were within normal limits.

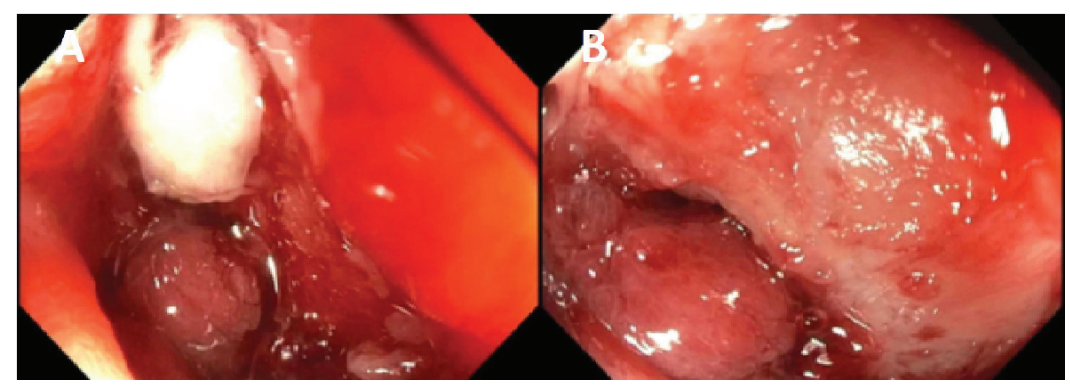

Figure 3. (A, B) Small bowel enteroscopy. A large ulcerated infiltrative mass, with bleeding upon contact and causing mechanical obstruction, was discovered in the fourth part of the duodenum. 


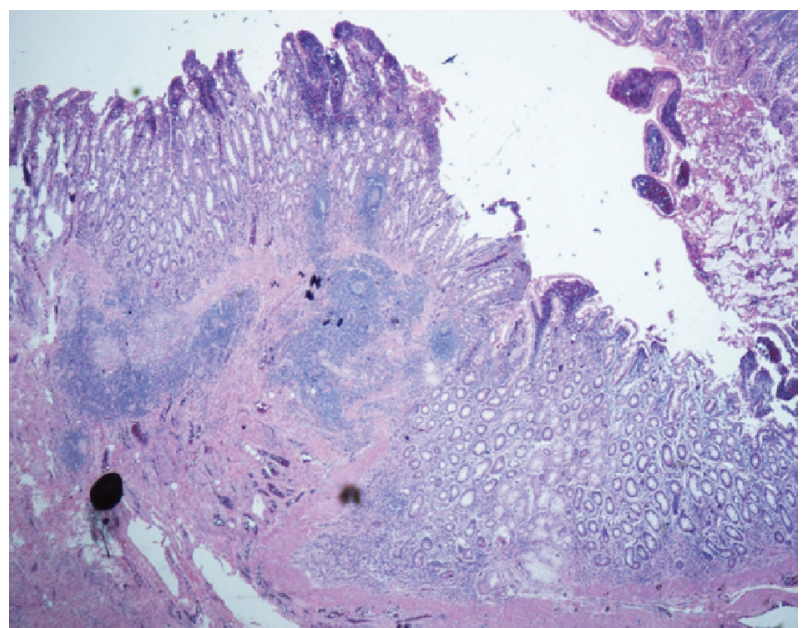

Figure 4. Histopathologic images of the patient's resected distal duodenum. The duodenal wall shows fissuring mucosal ulcer extending to the submucosa. There are poorly formed non-caseating granulomas. There is transmural inflammation seen in the form of lymphoid aggregates (H\&E stain).

The patient was diagnosed with Crohn's disease. He gradually improved after the surgical procedure with resolution of symptoms. Since then, he has demonstrated no further intestinal or extraintestinal manifestations of Crohn's disease, and, thus far, averted the need to start medical therapy to prevent the recurrence of Crohn's enteritis. As this patient had one isolated active lesion that was surgically resected, and his prior EGDs and colonoscopy did not reveal any additional lesions, he was not started on medical therapy. He has been symptomfree since then and is under observation.

\section{Discussion}

Crohn's disease comprises a wide array of clinical presentations. The spectrum of disease is diverse as a result of dissimilar phenotypes that cause distinctive symptoms and because the condition can involve almost any part of the digestive tract. Symptoms result from the transmural involvement of the gastrointestinal tract and include fistulas, phlegmon, abscesses, perianal disease, and malabsorption, as well as systemic symptoms and extraintestinal manifestations, such as arthritis or arthropathy, eye involvement, skin disorders, biliary tract involvement, and renal stones. Moreover, Crohn's disease varies considerably in terms of severity; it may be mild, moderate, or severe; it may be altogether tolerable or clinically disabling.

A subtype of Crohn's disease, gastroduodenal Crohn's disease, is observed in up to 15 percent of patients, many of whom present with upper abdominal pain, nausea, and/or postprandial vomiting [3]. The clinical features of this subtype often parallel those of peptic ulcer disease or gastric outlet obstruction. As this patient was recently diagnosed with $H$. pylori infection, another clinically significant differential diagnosis is peptic duodenitis, ulceration, and scarring, and its associated duodenal obstruction. Our patient had isolated involvement of the duodenum (stricturing type) and no additional intestinal or extraintestinal manifestations, which is unusual, and likely contributed to his delayed diagnosis.

Such patients with upper gastrointestinal tract involvement are at risk for small bowel obstruction, which results from recurrent inflammation which leads to strictures that cause mechanical obstruction or from stenotic areas with superimposed inflammation and spasm $[4,5]$. Undigested food can become impacted or intestinal adhesions can develop (particularly, in those who have had prior surgeries). Medical management with intravenous hydration, nasogastric suction, and parenteral nutrition is frequently efficacious, with a response observed within 24 to $48 \mathrm{~h}$ [5]. For patients who, on crosssectional imaging, have no evidence of proximal small bowel dilatation or long strictures $(>10 \mathrm{~cm})$, initial medical management with parenteral glucocorticoids is recommended. Surgical management is reserved for those patients who do not respond to medical management or who have evidence of small bowel ischemia [6, 7]. In addition, mucosal erosions, bleeding, and perforation have been described $[8,9]$. Thus, detection of bowel obstruction has important clinical implications for surgical intervention, which may involve segmental resection.

In conclusion, the occurrence of strictures, and the subsequent development of small bowel obstruction, as complications of Crohn's disease remains a challenging clinical problem. Despite recent advances and updated guidelines in the management of Crohn's disease, obstructive and subocclusive symptoms continue to occur, predominantly in the fibrostenosing phenotype and particularly in the presence of clinically significant strictures [10]. Compounding this problem is the fact that no specific anti-fibrotic therapies are available. Although it is clear that surgical intervention is indicated for an obstructing stricture that fails to respond to medical management, the optimal clinical approach for Crohn's disease-associated intestinal obstruction remains somewhat controversial [11].

\section{Financial Support}

The authors declare that no financial support or grant support has been received for the preparation of this manuscript.

\section{Conflict of Interest}

The authors declare that they have no competing interests. The authors declare that they have no affiliations with or involvement in any organization or entity with any financial interest or non-financial interest (such as personal or professional relationships, affiliations, knowledge, or beliefs) in the subject matter discussed in this manuscript.

\section{Author Contributions}

AS conceived of the idea for the manuscript. JS designed and drafted the manuscript. DE, MR, JK, AS, and AB evaluated and critically revised the manuscript for important intellectual 
content. AS and AB provided the images.

\section{References}

1. Mekhjian HS, Switz DM, Melnyk CS, Rankin GB, Brooks RK. Clinical features and natural history of Crohn's disease. Gastroenterology. 1979;77(4 Pt 2):898-906.

2. Freeman HJ. Natural history and long-term clinical course of Crohn's disease. World J Gastroenterol. 2014;20(1):3136.

3. Annunziata ML, Caviglia R, Papparella LG, Cicala M. Upper gastrointestinal involvement of Crohn's disease: a prospective study on the role of upper endoscopy in the diagnostic work-up. Dig Dis Sci. 2012;57(6):1618-1623.

4. Gore RM, Balthazar EJ, Ghahremani GG, Miller FH. CT features of ulcerative colitis and Crohn's disease. AJR Am J Roentgenol. 1996;167(1):3-15.

5. Rieder F, Zimmermann EM, Remzi FH, Sandborn WJ. Crohn's disease complicated by strictures: a systematic review. Gut. 2013;62(7):1072-1084.
6. Strong S, Steele SR, Boutrous M, Bordineau L, Chun J, Stewart DB, Vogel J, et al. Clinical practice guideline for the surgical management of Crohn's disease. Dis Colon Rectum. 2015;58(11):1021-1036.

7. Jones DW, Finlayson SR. Trends in surgery for Crohn's disease in the era of infliximab. Ann Surg. 2010;252(2):307312.

8. Yuan JG, Sachar DB, Koganei K, Greenstein AJ. Enterolithiasis, refractory anemia, and strictures of Crohn's disease. J Clin Gastroenterol. 1994;18(2):105-108.

9. Muthukumarasamy G, Nairn ER, McMillan I. Enterolith and small bowel perforation in Crohn's disease. Inflamm Bowel Dis. 2011;17(9):E126-127.

10. Vermeire S, Van Assche G, Rutgeerts P. Classification of inflammatory bowel disease: the old and the new. Curr Opin Gastroenterol. 2012;28(4):321-326.

11. Dignass A, Van Assche G, Lindsay JO, Lemann M, Soderholm J, Colombel JF, Danese S, et al. The second European evidence-based Consensus on the diagnosis and management of Crohn's disease: Current management. J Crohns Colitis. 2010;4(1):28-62. 